\title{
Genetic parameters for hoof health traits estimated with linear and threshold models using alternative cohorts
}

\author{
F. Malchiodi, ${ }^{1}{ }^{1}$ A. Koeck, ${ }^{*}$ S. Mason,† A. M. Christen,‡ D. F. Kelton,§ F. S. Schenkel, ${ }^{*}$ and F. Miglior \# \\ ${ }^{*}$ Centre for Genetic Improvement of Livestock, Department of Animal Biosciences, University of Guelph, Guelph, N1G 2W1 Ontario, Canada \\ †Department of Production Animal Health, University of Calgary, Calgary, T2N 1N4 Alberta, Canada \\ ‡alacta, Sainte-Anne-De-Bellevue, Quebec H9X 3R4, Canada \\ $\S$ Department of Population Medicine, Ontario Veterinary College, University of Guelph, Guelph, N1G 2W1 Ontario, Canada \\ \#Canadian Dairy Network, Guelph, N1K 1E5 Ontario, Canada
}

\begin{abstract}
A national genetic evaluation program for hoof health could be achieved by using hoof lesion data collected directly by hoof trimmers. However, not all cows in the herds during the trimming period are always presented to the hoof trimmer. This preselection process may not be completely random, leading to erroneous estimations of the prevalence of hoof lesions in the herd and inaccuracies in the genetic evaluation. The main objective of this study was to estimate genetic parameters for individual hoof lesions in Canadian Holsteins by using an alternative cohort to consider all cows in the herd during the period of the hoof trimming sessions, including those that were not examined by the trimmer over the entire lactation. A second objective was to compare the estimated heritabilities and breeding values for resistance to hoof lesions obtained with threshold and linear models. Data were recorded by 23 hoof trimmers serving 521 herds located in Alberta, British Columbia, and Ontario. A total of 73,559 hoof-trimming records from 53,654 cows were collected between 2009 and 2012. Hoof lesions included in the analysis were digital dermatitis, interdigital dermatitis, interdigital hyperplasia, sole hemorrhage, sole ulcer, toe ulcer, and white line disease. All variables were analyzed as binary traits, as the presence or the absence of the lesions, using a threshold and a linear animal model. Two different cohorts were created: Cohort 1 , which included only cows presented to hoof trimmers, and Cohort 2, which included all cows present in the herd at the time of hoof trimmer visit. Using a threshold model, heritabilities on the observed scale ranged from 0.01 to 0.08 for Cohort 1 and from 0.01 to 0.06 for Cohort 2 . Heritabilities estimated with the linear model ranged from 0.01 to 0.07 for Cohort 1 and from 0.01 to 0.05 for
\end{abstract}

Received June 3, 2016.

Accepted December 5, 2016.

${ }^{1}$ Corresponding author: fmalchio@uoguelph.ca
Cohort 2. Despite a low heritability, the distribution of the sire breeding values showed large and exploitable variation among sires. Higher breeding values for hoof lesion resistance corresponded to sires with a higher prevalence of healthy daughters. The rank correlations between estimated breeding values ranged from 0.96 to 0.99 when predicted using either one of the 2 cohorts and from 0.94 to 0.99 when predicted using either a threshold or a linear model.

Key words: hoof lesion, threshold model, genetic parameter

\section{INTRODUCTION}

Several studies in Europe and North America have reported a high prevalence of hoof lesions in dairy herds, with 40 to $70 \%$ of cows having at least 1 type of hoof lesion (Manske et al., 2002; Buch et al., 2011; Chapinal et al., 2013). The large prevalence of hoof lesions represents a significant welfare problem, but it also poses an economic loss for farmers because of the costs associated with treating the lesion and with decreased cow performance. The presence of hoof lesions has been reported to reduce milk production (Ettema et al., 2007; Amory et al., 2008) and to be associated with a lower reproductive performance of cows (Hultgren et al., 2004). Therefore, reducing the incidence of hoof lesions is important. This reduction can be achieved through improvement in management practices and possibly through genetic selection. Previous studies of Canadian Holsteins showed that hoof lesion data collected by hoof trimmers can be used for genetic evaluation of hoof health (Chapinal et al., 2013). However, not all cows in the herds are usually presented to the hoof trimmer during the trimming period, and the reasons for selection are usually not reported. Whether a cow is presented to the hoof trimmer is unlikely to be random because cows showing hoof problems may be selected for examination over cows that appear to be healthy. This selection could result in erroneous estimations of 
the prevalence of hoof lesions on the farm and may also affect the genetic evaluation of those traits. Considering only herds with a high percentage of trimmed cows may lead to bias through selecting herds with a specific management strategy or herds with more lame cows (van der Spek et al., 2013). Van der Spek et al. (2013) reported that the selection of cows for trimming does not affect the heritability. However, the result may change with the prevalence of nontrimmed cows in the population.

Threshold models are theoretically better for use when binary traits, such as the presence or absence of a hoof lesion, are considered. Moreover, they offer a better comparison between traits that have different incidences because linear models are known to be frequency dependent when applied to binary traits (Gianola, 1982). However, linear models are computationally more practical for the scale and scope of routine genetic evaluations. Furthermore, previous work did not show relevant differences between sire rankings for the 2 models (Weller et al., 1988; Hagger and Hofer, 1990).

The objectives of this study were (1) to estimate genetic parameters for individual hoof lesions in Canadian Holsteins using an alternative cohort to consider all cows in the herd during the period of the hoof trimming sessions, including those that were not examined by the hoof trimmer over the entire lactation; and (2) to compare the estimated heritabilities and breeding values for resistance to hoof lesions obtained with threshold and linear models.

\section{MATERIALS AND METHODS}

\section{Data}

Hoof lesions were recorded in 521 herds located in Alberta (from June 2009 to November 2012), British Columbia (from October 2010 to March 2012), and Ontario (from August 2011 to July 2012). Data were recorded by 23 trimmers trained to use a rugged touchscreen computerized lesion recording system (Hoof Supervisor, Dresser, WI) based on lesion descriptions proposed by the International Lameness Committee, a global collaboration of researchers, veterinarians, academics, and hoof trimming professionals. Hoof trimmers were also provided with a lesion severity scoring guide containing example photos of each lesion created by Alberta hoof trimmers to facilitate the correct denomination of the lesion and to more consistently score lesion severity (Alberta Dairy Hoof Health Project, 2014). Hoof lesions included in the analysis were digital dermatitis (DD), interdigital dermatitis (ID), interdigital hyperplasia $(\mathbf{I H})$, sole hemorrhage (SH), sole ulcer (SU), toe ulcer (TU), and white line diseases (WL). Due to very low frequency, corkscrew claw, heel horn erosion, foot rot, axial fissure, vertical fissure, horizontal fissure, thin sole, and unidentified lesions were combined into a single group denominated "other lesions" and were considered only for descriptive purpose. Finally, a variable was created that included the absence or the presence of any of the hoof lesions previously described. All traits were coded as binary variables $(0 ; 1)$, where 1 was assigned to the presence of a lesion. The initial data set included 126,621 hooftrimming records. Trimming sessions with missing calving date $(9.2 \%)$ and trimming data recorded after $500 \mathrm{~d}$ postpartum $(2.6 \%)$ were deleted. If the trimming date did not correspond to the period in which the cow was in the herd $(1.3 \%)$, the trimming session was deleted. A minimum of 10 records per hoof trimming session per herd were required to include data for any particular day in the analysis $(1.3 \%$ of the records were deleted). The majority of the cows $(65 \%)$ were trimmed only once during the lactation; therefore, only the first hoof trimming session was included in the analyses, as previously described by Chapinal et al. (2013). Finally, 2 different cohorts were created. The first cohort (Cohort 1) included only data from cows that had been visited at least once by the trimmer during the course of lactation. In the second cohort (Cohort 2), all cows that were in a given herd during the trimming period were included in the analyses, including cows that did not have any hoof data during the lactation. In such cases, the trimming session date was replaced with the first trimming session available in that herd during the lactation, and a value of 0 was assigned to all hoof traits for that trimming session. The final data set consisted of 75,559 hoof-trimming records from 53,654 cows when only trimmed cows were considered (Cohort 1) and 104,446 records from 70,394 cows in Cohort 2. The final pedigree files for the first and the second data set contained 196,879 animals and 230,267 animals, respectively, and included 7 previous generations.

\section{Models}

When linear models are applied to binary traits, the heritability estimates are frequency dependent (Gianola, 1982). Including the nontrimmed cows in the data set (Cohort 2) changed the incidence of each trait, decreasing the prevalence of each lesion. To account for this decrease and to better compare the 2 cohorts, a threshold approach was applied. Although threshold models are more appropriate to analyze binary traits, linear models are easier to implement for the scale and scope of a routine genetic evaluation. Therefore, data were analyzed in the DMU package (Madsen and 
Jensen, 2008), using either a univariate linear mixed model in the AI-REML procedure or a univariate generalized linear mixed model (GLMM) with a probit link function: $\mathrm{E}\left(y_{m}\right)=\Phi\left(\lambda_{m}\right)$, where $\mathrm{E}\left(y_{m}\right)$ is the expected value for the $m$ th observation ( 0 or 1 ), $\Phi$ is the normal cumulative density function, and $\lambda_{m}$ is $m$ th fitted value on the underlying normal scale.

A linear model similar to that previously described by Chapinal et al. (2013) was applied to all lesion traits:

$$
\begin{aligned}
Y_{i j k l m n}=\mu+ & H D_{i}+T R_{j}+P_{k}+L_{l}+a_{m} \\
& +p e_{n}+e_{i j k l m n},
\end{aligned}
$$

where $Y_{i j k l m n}$ is the observation for one of the lesion traits $(0=$ nonaffected or $1=$ affected $), \mu$ is the overall mean, $H D_{i}$ is the fixed effect of herd-date of hoof trimming ( $i=1$ to 3,086), $T R_{j}$ is the fixed effect of hoof trimmer $(j=1$ to 23$), P_{k}$ is the fixed effect of parity at trimming ( $k=1$ to 7 and more), $L_{l}$ is the fixed effect of stage of lactation at trimming ( $l=1$ to 16 ; class 1 : $0-30$ $\mathrm{d}$, class 2: 31-60 d, ..., class 15: 421-450 d, and class 16: $451-500 \mathrm{~d}$ after calving), $a_{m}$ is the random additive genetic animal effect ( $m=1$ to 196,879 for Cohort $1 ; m=1$ to 230,367 for Cohort 2), $p e_{n}$ is the random permanent environmental effect $(n=1$ to 53,654 for Cohort $1 ; n=1$ to 70,394 for Cohort 2), and $e_{i j k l m n}$ is the random error term.

Random effects were assumed to be normally distributed, with means equal to zero and covariance structure equal to

$$
\operatorname{Var}\left[\begin{array}{c}
\mathbf{a} \\
\mathbf{p e} \\
\mathbf{e}
\end{array}\right]=\left[\begin{array}{ccc}
\mathbf{A} \sigma_{a}^{2} & 0 & 0 \\
0 & \mathbf{I} \sigma_{p e}^{2} & 0 \\
0 & 0 & \mathbf{I} \sigma_{e}^{2}
\end{array}\right]
$$

where $\sigma_{a}^{2}$ is the additive genetic variance, $\sigma_{p e}^{2}$ is the permanent environmental variance, and $\sigma_{e}^{2}$ is the residual variance; $\mathbf{A}$ is the additive genetic relationship matrix; and $\mathbf{I}$ is an identity matrix.

Subsequently, bivariate linear animal models were used to estimate the genetic and phenotypic correlations between different hoof lesions. Random effects were assumed to be normally distributed, with means equal to zero and covariance structure in the bivariate analyses as follows:

$$
\operatorname{Var}\left[\begin{array}{c}
\mathbf{a} \\
\mathbf{p e} \\
\mathbf{e}
\end{array}\right]=\left[\begin{array}{ccc}
\mathbf{G}_{0} \otimes \mathbf{A} & 0 & 0 \\
0 & \mathbf{P E}_{0} \otimes \mathbf{I} & 0 \\
0 & 0 & \mathbf{R}_{0} \otimes \mathbf{I}
\end{array}\right]
$$

where $\mathbf{G}_{0}$ is the genetic covariance $(2 \times 2)$ matrix between traits due to animal additive genetic effects; $\mathbf{P E}_{0}$ is the covariance $(2 \times 2)$ matrix between traits due to permanent environmental effects; $\mathbf{R}_{0}$ is the residual covariance $(2 \times 2)$ matrix between traits; $\otimes$ is the Kronecker product; $\mathbf{A}$ is the additive genetic relationship matrix, and $\mathbf{I}$ is an identity matrix.

To compare the results from the threshold and the linear model, the heritabilities estimated with the threshold model were transformed into the observed scale through the Dempster and Lerner (1950) method:

$$
h_{\mathrm{obs}}^{2}=\frac{h_{\mathrm{und}}^{2} \times\left(z_{\mathrm{ord}}\right)^{2}}{p \times(1-p)},
$$

where $h_{\mathrm{obs}}^{2}$ is the heritability on the observed scale, $h_{\mathrm{und}}^{2}$ is the heritability on the underlying scale estimated with the threshold model, $z_{\text {ord }}$ is the ordinate height of the normal distribution at the threshold point corresponding to $p$, and $p$ is the prevalence of the hoof lesion.

Finally, Spearman rank correlations between the EBV predicted using the 2 different cohorts (Cohort 1 vs. Cohort 2) and between the EBV predicted with the 2 statistical approaches (threshold vs. linear model) were calculated using the CORR procedure of SAS (SAS Institute Inc., 2013).

\section{RESULTS AND DISCUSSION}

\section{Prevalence of Hoof Lesions}

The prevalence of hoof lesions across parities is reported in Tables 1 and 2 for Cohort 1 and Cohort 2, respectively. Overall, $37.8 \%$ of cows presented to the hoof trimmers had at least 1 type of hoof lesion. The more common lesion was DD, with $20 \%$ of cows presented to the hoof trimmers being affected, followed by SU $(7 \%)$ and WL (5.4\%). When the second cohort was considered, the prevalence of cows with at least 1 hoof lesion was $26.6 \%$. As mentioned before, the process by which a cow is presented to the hoof trimmers is not completely random because cows with hoof lesions are likely to be preferentially presented to the hoof trimmers. As a consequence, the prevalence of hoof lesions calculated by data recorded directly from hoof trimmers can be overestimated. However, the prevalence of lesions in Cohort 2 may be underestimated because some of the cows that were considered healthy may have had undetected lesions. Generally, the prevalence of each hoof lesion in Cohort 2 (Table 2) was 29 to $31 \%$ lower than the prevalence reported in Cohort 1 (Table 1). This value corresponded to the proportion 
Table 1. Percentage of hoof trimming records with hoof lesions, including only the first trimming session of the lactation among cows that have been visited at least once by the trimmer during the lactation (Cohort 1), and stratified by parity

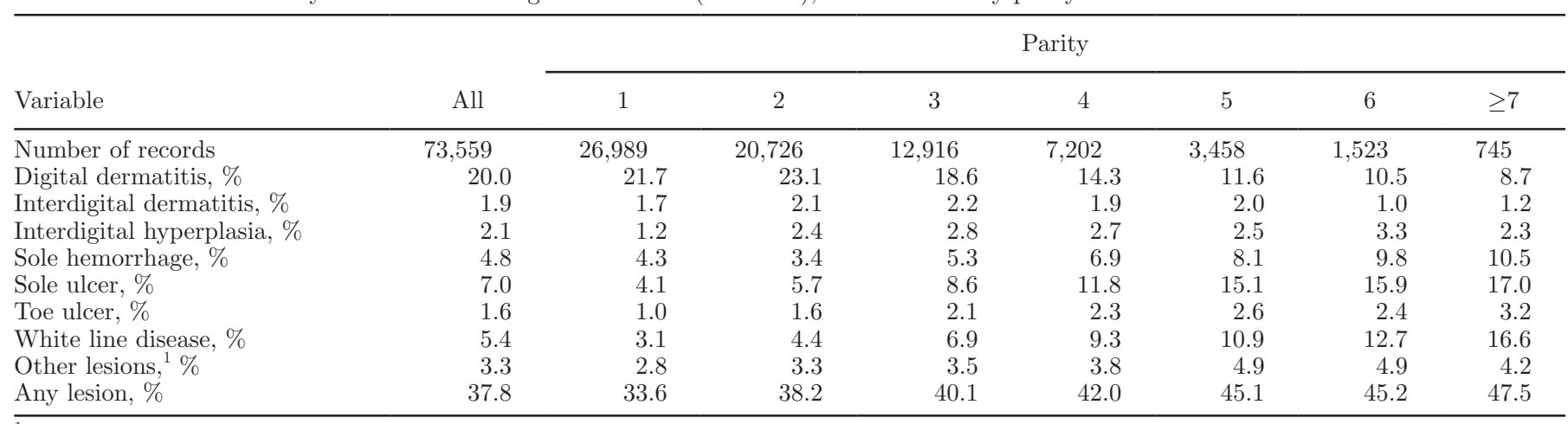

${ }^{1}$ Corkscrew claw, heel horn erosion, foot rot, axial fissure, vertical fissure, horizontal fissure, thin sole, and unidentified lesions.

of nontrimmed cows that were added to the second data set $(30 \%$ of cows of Cohort 2 were cows without a trimming record). In a previous study, van der Spek et al. (2013) reported that the prevalence of hoof lesions decreased by 58 to $59 \%$ when untrimmed cows were included in the analyses. In that study, the proportion of cows without a trimming record was $59 \%$. The percentage of cows trimmed per herd was lower than $35 \%$ in 386 herds, between 35 and $69 \%$ in 207 herds, and equal or higher than $70 \%$ in 62 herds (van der Spek et al., 2013). In the present study, only 25 herds had less than $35 \%$ of cows trimmed, 145 herds had 35 to $69 \%$ of cows trimmed, and 351 herds had $70 \%$ or more cows trimmed.

Generally, the prevalence of DD decreased across parity number, whereas the prevalence of $\mathrm{SH}, \mathrm{SU}, \mathrm{TU}$, and WL increased. These results are in agreement with Koenig et al. (2005), who reported an increase in the incidence of SU and wall disorders with increasing parities and a higher prevalence of DD among first parity cows. Similarly, Chapinal et al. (2013) reported an overall increase in horn lesions (SU, TU, SH, and WL) and a decrease in infectious or partly infectious lesions (DD, ID, foot rot, and heel erosion) across parity. The increase in horn lesions can be partially explained by the presence of irreversible hoof lesions, so that the prevalence increases with parity (Koenig et al., 2005). On the other hand, the increase in immunity with age could partially explain the decrease of infectious lesions (Chapinal et al., 2013). However, van der Linde et al. (2010) reported an increase over parity in the prevalence of ID, also an infectious lesion, whereas a decrease in the prevalence of DD was reported. Therefore, other mechanisms may be involved in the decrease in DD within lactation. For example, the decrease in infectious lesions with parity could be due to an increase in the thickness of the skin, which makes it more difficult for pathogens to enter and cause the infection, and this growth may be less pronounced in the interdigital space than in the digital space.

The prevalence of lesions was comparable to values previously reported in Canada (Chapinal et al., 2013;

Table 2. Percentage of hoof-trimming records with hoof lesions, including only the first trimming session of the lactation, among all cows that were in the herds during the trimming period (Cohort 2$),{ }^{1}$ and stratified by parity

\begin{tabular}{|c|c|c|c|c|c|c|c|c|}
\hline Variable & All & \multicolumn{7}{|c|}{ Parity } \\
\hline Interdigital dermatitis, $\%$ & 1.3 & 1.3 & 1.6 & 1.5 & 1.4 & 1.4 & 0.7 & 0.9 \\
\hline Interdigital hyperplasia, \% & 1.4 & 0.9 & 1.7 & 2.0 & 2.0 & 1.7 & 2.4 & 1.7 \\
\hline Sole hemorrhage, \% & 3.4 & 3.2 & 2.5 & 3.8 & 5.0 & 5.7 & 7.0 & 7.6 \\
\hline White line disease, $\%$ & 3.8 & 2.3 & 3.2 & 5.0 & 6.8 & 7.6 & 9.1 & 12.1 \\
\hline Other lesions, ${ }^{2} \%$ & 2.3 & 2.1 & 2.4 & 2.5 & 2.8 & 3.4 & 3.5 & 3.0 \\
\hline Any lesion, \% & 26.6 & 25.0 & 27.7 & 28.7 & 30.4 & 31.5 & 32.4 & 34.6 \\
\hline
\end{tabular}

${ }^{1}$ For cows that did not have any hoof data during the lactation, a value of 0 was assigned to all hoof traits for the first trimming session available in the herd during the lactation.

${ }^{2}$ Corkscrew claw, heel horn erosion, foot rot, axial fissure, vertical fissure, horizontal fissure, thin sole, and unidentified lesions. 
Solano et al., 2016). The overall presence of lesions was lower compared with the values reported in other studies conducted in Europe (Manske et al., 2002; Ettema et al., 2007; van der Linde et al., 2010). Some of the observed differences could be due to the way in which data were analyzed. In contrast with the current study, in which only first hoof trimming sessions were included in the analyses, the aforementioned studies considered all repeated observations (within and across lactations). However, differences in hoof lesion prevalence among countries could be the consequence of other factors, such as farm size, housing system, and access to pasture. The average herd size in this study was 122 cows for Alberta, 114 cows for British Columbia, and 64 cows for Ontario. These sizes were similar to those reported in 2012 by the Canadian Dairy Information Centre for these provinces $(152,138$, and 78 cows, respectively). Solano et al., (2016) also found that the type of housing system had an effect on the prevalence of hoof lesions, with cows housed in deep-bedded packs being the least affected. According to the Canadian Dairy Information Centre in 2012, the majority of the farms in Alberta and British Columbia used freestall barns (89.6 and $84.5 \%$ of the farms, respectively). However, in Ontario a higher proportion of farms used tie-stall barns. In 2012, 70\% of herds in Ontario were housed in tiestall barns, while only $26.7 \%$ were reported to be in freestall barns. Cramer et al. (2008) reported a higher prevalence of lesions for freestall compared with tiestall barns in Ontario (46.8 and $25.7 \%$ of cows affected by at least 1 lesion, respectively). The higher prevalence of lesions among cows in freestall barns was reported for both infectious and horn lesions. In the present study, the type of housing system was not available, so it was not possible to investigate its effect. However, the prevalence of lesions was not distinctly higher in Ontario compared with Alberta and British Columbia, which use predominantly freestall barns. The prevalence of cows with at least 1 lesion was $34 \%$ in Alberta, $48 \%$ in British Columbia, and 39\% in Ontario.

\section{Heritabilities of Hoof Lesions}

When only hoof trimmed cows were included in the analysis and a threshold model was applied, estimated heritabilities of hoof lesions ranged from 0.06 to 0.19 on the underlying scale (Table 3). Similar heritabilities were reported for German Holstein and Norwegian Red cows for DD, SU, and WL, when estimated using a threshold model $(0.13,0.17$, and 0.10 , respectively, Swalve et al., 2008; and 0.20, 0.18, 0.06, respectively, Ødegård et al., 2013). Swalve et al. (2008) also reported a higher heritability estimate for ID (0.20). In the current study, the estimated heritabilities for ID were 0.13 and 0.12 for Cohort 1 and Cohort 2, respectively. In the study of Swalve et al. (2008), however, the prevalence of ID was much higher than in our study (11.9\% of affected cows). A similar heritability for DD (0.13) was reported for Finnish Holsteins, when a logistic regression model was applied (Häggman and Juga, 2013). The estimated heritability reported by Häggman and Juga (2013) for SU (0.08) was similar to the heritability estimated in the present study when all cows were included in the analysis (0.07, Cohort 2$)$. In the same study, lower heritability estimates were reported for ID and $\mathrm{SH}$ (0.03 and 0.02, respectively).

The heritabilities estimated with the linear animal model ranged from 0.01 to 0.07 , when only hoof-trimmed cows were included in the analysis (Cohort 1). Similar heritabilities for hoof lesions have been reported by van der Linde et al. (2010) for Dutch Holstein-Friesians. Chapinal et al. (2013) reported a heritability of 0.08 for infectious lesions (analyzed together as 1 trait) for Canadian Holsteins. In the current study, DD and ID

Table 3. Heritability (SE) on the underlying scale $\left(\mathrm{h}^{2}{ }_{\text {und }}\right)$ and heritability transformed to the observed scale by the Dempster and Lerner (1950) method $\left(\mathrm{h}^{2}{ }_{\text {obs-t }}\right)$ estimated using a univariate probit threshold animal model, and heritability on the observed scale $\left(\mathrm{h}^{2}{ }_{\text {obs- }-1}\right)$ estimated using a univariate linear animal model when only hoof-trimmed cows were considered (Cohort 1), and when all cows present in the herd at time of hoof trimmer visit were included (Cohort 2$)^{1}$

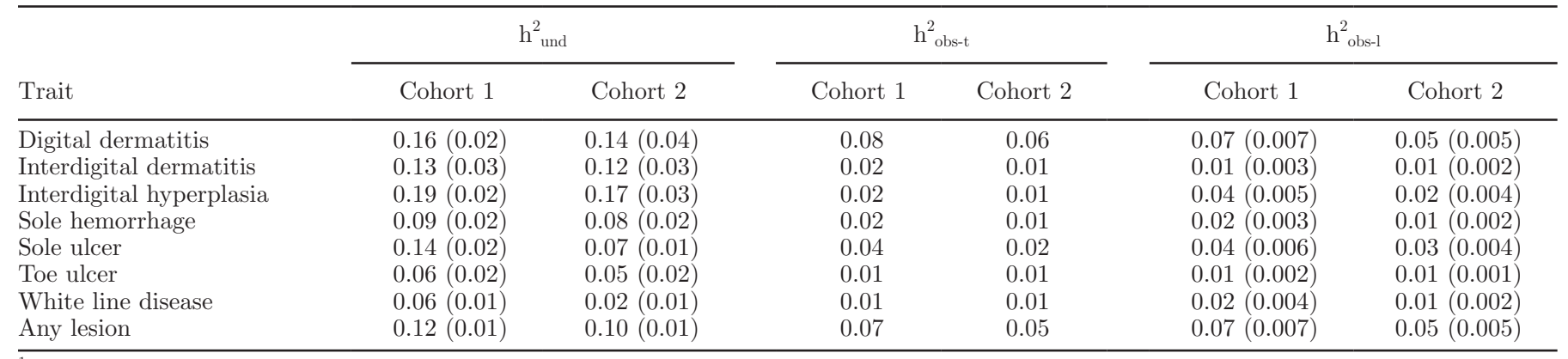

${ }^{1}$ For cows that did not have any hoof data during the lactation, a value of 0 was assigned to all hoof traits for the first trimming session available in the herd during the lactation. 
were considered as separate traits, and their heritabilities were 0.07 and 0.01 , respectively. The difference in heritability estimates for these 2 infectious lesions was likely due to the large difference in their prevalence. Moreover, considering the heritability estimated with threshold model, DD and ID showed more similar heritability estimates ( 0.16 and 0.13 , respectively). Similarly, horn lesions (analyzed as 1 trait) were previously reported to have an estimated heritability of 0.03 (Chapinal et al., 2013), ranging in the current study from 0.01 to 0.04 for different horn lesion types. Koenig et al. (2005) obtained similar results for DD, using a logistic approach, and estimated a heritability of 0.07 . In the same study, slightly higher heritabilities were shown for SU and IH (0.09 and 0.10, respectively). Other studies (van der Linde et al., 2010; van der Spek et al., 2013) reported higher heritabilities for IH (0.13 and 0.14, respectively) in Dutch Holstein-Friesian cows. In both studies, this lesion affected more cows than in the current study ( 5 and $8.7 \%$ of cows affected by $\mathrm{IH}$, respectively). However, the difference in the heritability cannot be explained only by the difference between the incidences of the lesions in the 2 studies. Even considering the heritability on the underlying scale, the value reported by van der Spek et al. (2013) was higher than the one found in our study (0.43 and 0.19 , respectively). When transformed to the observed scale, the heritabilities estimated with the threshold model were very similar to the heritabilities estimated with the linear model (Table 3).

The estimated heritability was slightly lower when Cohort 2 was analyzed (Table 3 ). However, when the heritability on the underlying scale was considered, this difference was reduced, indicating that the aforementioned differences were mostly related to a lower frequency of hoof lesions in Cohort 2. The greatest difference between heritabilities estimated with the threshold model for the 2 cohorts was found for WL (0.06 and 0.02 for Cohort 1 and Cohort 2, respectively). In agreement with findings reported by van der Spek et al. (2013), these results suggest that the preselection process of cows for trimming does not have a severe effect on the estimated heritabilities.

\section{Genetic and Phenotypic Correlations Among Hoof Lesions}

Genetic correlations between individual hoof lesions were very similar across the 2 cohorts (Tables 4 and 5). Infectious lesions showed moderate genetic correlations with IH. Similarly, van der Spek et al. (2013) reported a correlation of 0.66 between IH and dermatitis erosion, which is a group that combined DD, ID, and heel horn erosion. Weak genetic correlations were found between infectious and horn lesions, with the exception of WL, that showed moderate negative correlations with DD and ID $(-0.30$ and -0.41 , respectively, for Cohort 1; -0.23 and -0.26 , respectively, for Cohort 2). As in other studies (Buch et al., 2011; van der Spek et al., 2013), these results suggest that the genetic background for infectious and horn lesions is probably different. Genetic correlations among horn lesions were moderate to strong. The strongest correlations were between SU and SH $(0.80$ and 0.83 for Cohort 1 and Cohort 2, respectively). Similar results were reported by van der Spek et al. (2013; genetic correlation of 0.90 between SU and SH), whereas Häggman and Juga (2013) and Johansson et al. (2011) estimated a lower correlation between these 2 horn lesions (0.38 and 0.39 , respectively). No significant genetic correlation was found between $\mathrm{SH}$ and TU in the current study. The genetic correlation with the overall presence of a lesion was stronger for the more prevalent lesions (DD and SU) and moderate for the other hoof lesions. These results, together with

Table 4. Genetic correlations (above the diagonal; SE in parentheses) and phenotypic correlations (below the diagonal; SE not available) from bivariate linear animal models when only cows that have been visited at least once by the trimmer during the lactation were included (Cohort 1)

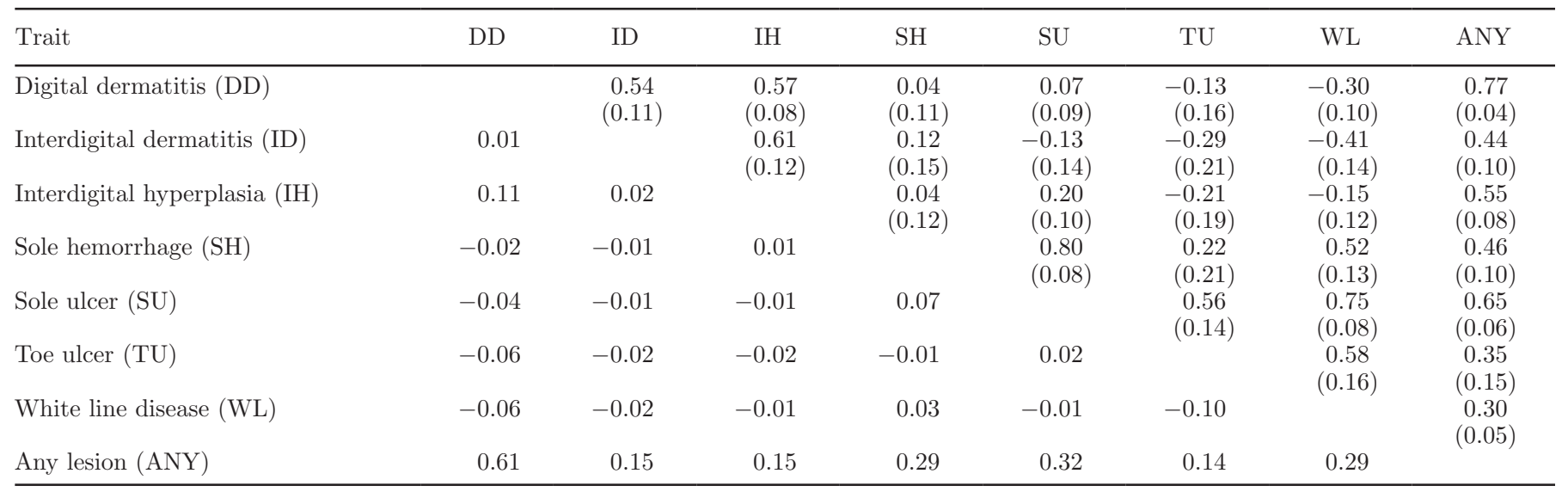


Table 5. Genetic correlations (above the diagonal; SE in parentheses), and phenotypic correlations (below the diagonal; SE not available) from bivariate linear animal models when all cows present in the herd at time of hoof trimmer visit were included (Cohort 2$)^{1}$

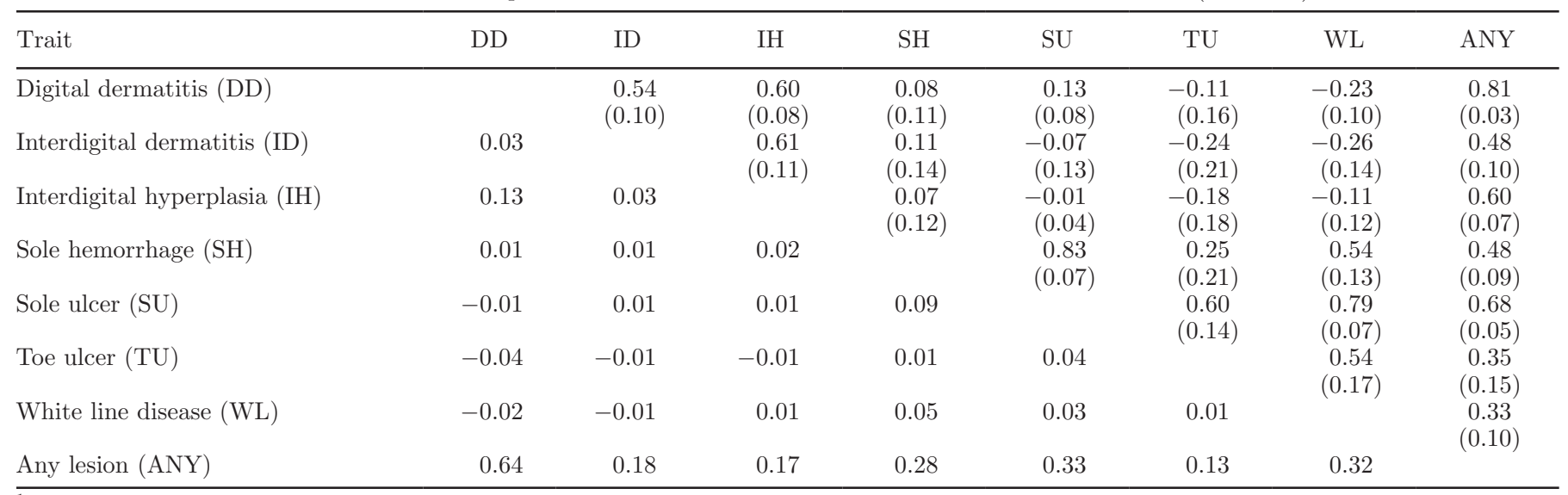

${ }^{1}$ For cows that did not have any hoof data during the lactation, a value of 0 was assigned to all hoof traits for the first trimming session available in the herd during the lactation.

the genetic correlations between individual hoof lesions previously reported, suggest that combining several hoof lesions in a genetic selection plan will not be ideal, and lesions should be considered individually according to their etiology and pathogenesis.

Phenotypic correlations between hoof lesions were very low, with estimates ranging from -0.10 (between WL and TU) to 0.11 (between DD and $\mathrm{IH}$ ) for Cohort 1 and from -0.04 (between DD and TU) to 0.13 (between DD and $\mathrm{IH}$ ) for Cohort 2. In a previous study, Häggman and Juga (2013) reported low negative phenotypic correlations between hoof lesions, ranging from -0.24 (between $\mathrm{SH}$ and white line separation) to -0.06 (between heel horn erosion and corkscrew hoof). The phenotypic correlations reported by van der Spek et al. (2013) were generally weak, ranging from -0.02 (between IH and $\mathrm{SH}$ ) to 0.11 (between double sole and WL). The phenotypic correlations between the general presence of at least 1 hoof lesion and the single hoof lesions were low to moderate, ranging from 0.14 (with
TU) to 0.61 (with DD) in Cohort 1. Similarly, in Cohort 2, the strongest correlation was found between DD and the overall presence of hoof lesion (0.64), while a weak correlation was found with TU (0.13).

\section{Prediction of Breeding Values}

Estimated breeding values were reversed in sign, so that higher EBV corresponds to sires with higher number of healthy daughters (Table 6). Despite a low heritability, the distribution of the sire EBV showed significant variation among sires. Generally, the variation among sires was similar between the 2 cohorts. For DD, only $10 \%$ of daughters of the top $10 \%$ sires were affected, whereas $35.2 \%$ of daughters of the bottom $10 \%$ of sire were affected for Cohort 1 . For Cohort 2 , only $6.5 \%$ of daughters of the top $10 \%$ sires were affected, while $24.1 \%$ of daughters of the bottom $10 \%$ of sire were affected by DD. A good variability among sires has been previously reported by Chapinal et al.

Table 6. Percentage of daughters with hoof lesions from all (average), the best ( $10 \%$ decile), and the worst (90\% decile) sires according to their EBV for resistance to hoof lesions predicted with a univariate probit threshold model

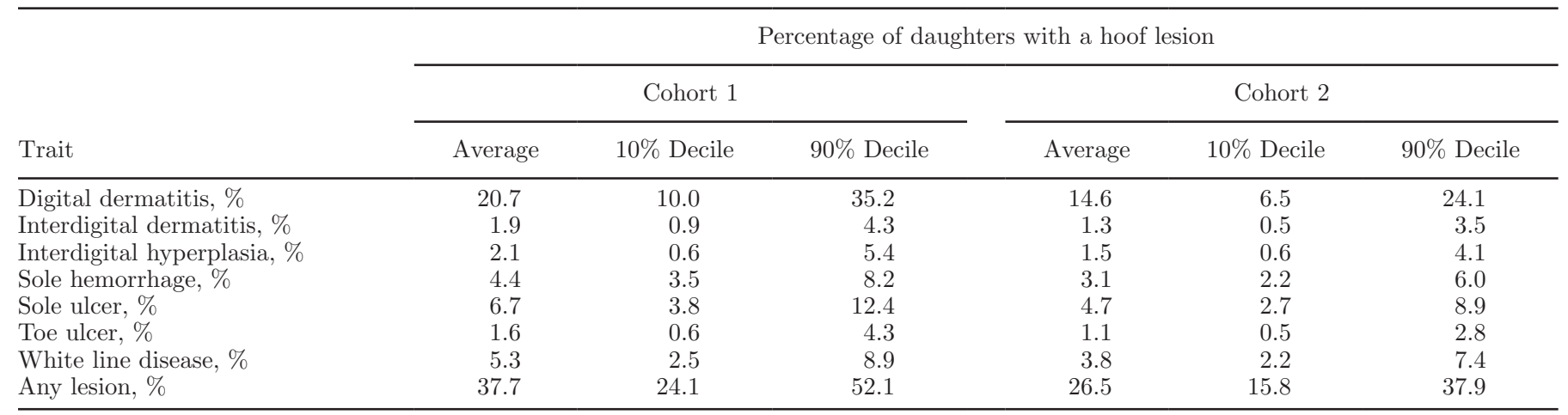


Table 7. Rank correlations between EBV predicted using 2 alternative cohorts ${ }^{1}$ within 2 statistical approaches, ${ }^{2}$ and rank correlations between the EBV predicted with the 2 statistical approaches within cohorts

\begin{tabular}{lccccc}
\hline & \multicolumn{2}{c}{ Cohort 1 and Cohort 2} & & \multicolumn{2}{c}{ Linear and Threshold } \\
\cline { 2 - 3 } \cline { 5 - 6 } Trait & Threshold & Linear & & Cohort 1 & Cohort 2 \\
\hline Digital dermatitis & 0.98 & 0.98 & & 0.99 & 0.99 \\
Interdigital dermatitis & 0.98 & 0.98 & & 0.97 & 0.96 \\
Interdigital hyperplasia & 0.99 & 0.99 & & 0.96 & 0.96 \\
Sole hemorrhage & 0.99 & 0.98 & & 0.97 & 0.96 \\
Sole ulcer & 0.97 & 0.98 & & 0.98 & 0.96 \\
Toe ulcer & 0.98 & 0.98 & & 0.98 & 0.98 \\
White line disease & 0.97 & 0.98 & & 0.99 & 0.99 \\
Any lesion & 0.96 & 0.96 & & 0.99 \\
\hline
\end{tabular}

${ }^{1}$ Cohort 1 = only hoof trimmed cows; Cohort 2 = all cows present in the herd at the time of hoof trimmer visit.

${ }^{2}$ Probit threshold animal model or linear animal model.

(2013) in a study on Canadian Holstein involving a smaller data set. The 10 sires with the highest EBV were reported to have $15 \%$ of daughters with infectious lesions, while $53 \%$ of the daughters of the 10 sires with the lowest EBV presented infectious lesions. The corresponding values for resistance to horn lesions were 7 and $24 \%$ for the 10 sires with the highest and lowest EBV, respectively. In the current study, SU was the most prevalent horn lesion; 3.8 and $2.7 \%$ of daughters of the top $10 \%$ of sires were affected for Cohort 1 and Cohort 2, respectively, whereas 12.4 and $8.9 \%$ of daughters of the bottom $10 \%$ of sires were affected for Cohort 1 and Cohort 2, respectively.

The rank correlations between Cohort 1 and Cohort 2 EBV were very high, ranging from 0.96 and 0.99 (Table 7). The rank correlations between EBV predicted with linear or threshold model ranged from 0.94 and 0.99 and were similar between Cohort 1 and Cohort 2. The strongest correlation of 0.99 was found for the lesion with the highest prevalence (DD and at least 1 lesion).

\section{CONCLUSIONS}

Although the heritabilities were low, an exploitable genetic variation for hoof lesions in Canadian Holstein population exists, and a possibility therefore exists for improvement through long-term direct genetic selection. The exclusion of nontrimmed cows from the analysis led to a probable overestimation of the prevalence of hoof lesions in dairy herds, but it does not seem to have affected the estimation of genetic parameters. Moreover, estimated heritabilities showed similar values when data were analyzed using either a threshold or a linear model and the corresponding EBV were highly correlated. This outcome suggests that linear models could be used for evaluating hoof lesions because they are computationally less demanding than threshold models.

\section{ACKNOWLEDGMENTS}

The authors are grateful to the hoof trimmers who participated in this study. This research was supported by a contribution from the Dairy Research Cluster Initiative (Dairy Farmers of Canada, Agriculture and Agri-Food Canada, the Canadian Dairy Network, and the Canadian Dairy Commission) and Alberta Milk (Edmonton, AB, Canada).

\section{REFERENCES}

Alberta Dairy Hoof Health Project. 2014. The Alberta dairy hoof health project: Lesion severity scoring guide. http://dairyhoofhealth. info/Lesion-Severity-Guide-v0.7.pdf.

Amory, J. R., Z. E. Barker, J. L. Wright, S. A. Mason, R. W. Blowey, and L. E. Green. 2008. Associations between sole ulcer, white line disease and digital dermatitis and the milk yield of 1824 dairy cows on 30 dairy cow farms in England and Wales from February 2003-November 2004. Prev. Vet. Med. 83:381-391.

Buch, L. H., A. C. Sørensen, J. Lassen, P. Berg, J. A. Eriksson, J. H. Jakobsen, and M. K. Sørensen. 2011. Hygiene-related and feedrelated hoof diseases show different patterns of genetic correlations to clinical mastitis and female fertility. J. Dairy Sci. 94:1540-1551.

Chapinal, N., A. Koeck, A. Sewalem, D. F. Kelton, S. Mason, G. Cramer, and F. Miglior. 2013. Genetic parameters for hoof lesions and their relationship with feet and leg traits in Canadian Holstein cows. J. Dairy Sci. 96:2596-2604.

Cramer, G., K. D. Lissemore, C. L. Guard, K. E. Leslie, and D. F. Kelton. 2008. Herd- and cow-level prevalence of foot lesions in Ontario dairy cattle. J. Dairy Sci. 91:3888-3895.

Dempster, E. R., and I. M. Lerner. 1950. Heritability of threshold characters. Genetics 35:212-236.

Ettema, J. F., N. Capion, and A. E. Hill. 2007. The association of hoof lesions at claw trimming with test-day milk yield in Danish Holstein. Prev. Vet. Med. 79:224-243.

Gianola, D. 1982. Theory and analysis of threshold characters. J. Anim. Sci. 54:1079-1096.

Hagger, C., and A. Hofer. 1990. Genetic analyses of calving traits in the Swiss Black and White, Braunvieh and Simmental breeds by REML and MAPP procedures. Livest. Prod. Sci. 24:93-107.

Häggman, J., and J. Juga. 2013. Genetic parameters for hoof disorders and feet and leg conformation traits in Finnish Holstein cows. J. Dairy Sci. 96:3319-3325.

Hultgren, J., T. Manske, and C. Bergsten. 2004. Associations of sole ulcer at claw trimming with reproductive performance, udder 
health, milk yield, and culling in Swedish dairy cattle. Prev. Vet. Med. 62:233-251.

Johansson, K., J. Å. Eriksson, U. S. Nielsen, J. Pösö, and G. P. Aamand. 2011. Genetic evaluation of claw health in Denmark, Finland and Sweden. Interbull Bull. 44:224-228.

Koenig, S., A. R. Sharifi, H. Wentrot, D. Landmann, M. Eise, and H. Simianer. 2005. Genetic parameters of claw and foot disorders estimated with logistic models. J. Dairy Sci. 88:3316-3325.

Madsen, P., and J. Jensen. 2008. A User's Guide to DMU: A Package for Analyzing Multivariate Mixed Models. Version 6, release 4.7. Danish Institute of Agricultural Sciences, Tjele, Denmark.

Manske, T., J. Hultgren, and C. Bergsten. 2002. Prevalence and interrelationships of hoof lesions and lameness in Swedish dairy cows. Prev. Vet. Med. 54:247-263.

Ødegård, C., M. Svendsen, and B. Heringstad. 2013. Genetic analyses of claw health in Norwegian Red cows. J. Dairy Sci. 96:7274-7283.

SAS Institute. 2013. SAS ${ }^{\circledR} 9.4$ Guide to Software Updates. SAS Institute Inc., Cary, NC.
Solano, L., H. W. Barkema, S. Mason, E. A. Pajor, S. J. LeBlanc, and K. Orsel. 2016. Prevalence and distribution of foot lesions in dairy cattle in Alberta, Canada. J. Dairy Sci. 99:6828-6841.

Swalve, H. H., H. Alkhoder, and R. Pijl. 2008. Estimates of breeding values for sires based on diagnoses recorded at hoof trimming: Relationships with EBV for conformation traits. Interbull Bull. 38:87-90.

van der Linde, C., G. De Jong, E. P. C. Koenen, and H. Eding. 2010. Claw health index for Dutch dairy cattle based on claw trimming and conformation data. J. Dairy Sci. 93:4883-4891.

van der Spek, D., J. A. M. van Arendonk, A. A. A. Vallée, and H. Bovenhuis. 2013. Genetic parameters for claw disorders and the effect of preselecting cows for trimming. J. Dairy Sci. 96:6070-6078.

Weller, J. I., I. Misztal, and D. Gianola. 1988. Genetic analysis of dystocia and calf mortality in Israeli-Holsteins by threshold and linear models. J. Dairy Sci. 71:2491-2501. 